\title{
Mechanism of dialkyl phthalates removal from aqueous solution using $\gamma$-cyclodextrin and starch based polyurethane polymer adsorbents
}

\author{
Chukwunonso Peter Okoli ${ }^{\mathrm{a}, 1}$, Gregory Olufemi Adewuyi ${ }^{\mathrm{b}}$, Qian Zhang ${ }^{\mathrm{a}}$, \\ Paul N. Diagboya ${ }^{\mathrm{b}, \mathrm{c}}$, Qingjun Guo ${ }^{\mathrm{a}, *}$ \\ a Centre for Environmental Remediation, Institute of Geographic Sciences and Natural Resources Research, Chinese Academy of Sciences, Beijing, PR China \\ ${ }^{\mathrm{b}}$ Analytical/Environmental Chemistry Unit, Department of Chemistry, University of Ibadan, Ibadan, Nigeria \\ ${ }^{\mathrm{c}}$ National Centre for Nanoscience and Nanotechnology, Chinese Academy of Sciences, Beijing, PR China
}

\section{A R T I C L E I N F O}

\section{Article history}

Received 4 May 2014

Received in revised form 25 July 2014

Accepted 11 August 2014

Available online 19 August 2014

\section{Keywords:}

Phthalates

Polyurethane polymer

$\gamma$-cyclodextrin

Starch

Adsorption

\begin{abstract}
A B S T R A C T
Phthalate esters have been known as potent endocrine disruptors and carcinogens; and their removal from water have been of considerable concern recently. In the present study, $\gamma$-cyclodextrin polyurethane polymer (GPP), $\gamma$-cyclodextrin/starch polyurethane copolymer (GSP), and starch polyurethane polymer (SPP) have been synthesized and characterized. Their adsorption efficiencies for the removal of dimethyl phthalate (DMP) and diethyl phthalate (DEP) from aqueous solutions were investigated. The characterization results showed the success of the synthesis. The isotherms were L-type, and both the Langmuir and Freundlich adsorption isotherm gave good fittings to the adsorption data. Adsorption mechanisms suggested that these adsorbents spontaneously adsorb phthalate molecules driven mainly by enthalpy change, and the adsorption process was attributed to multiple adsorbent-adsorbate interactions such as hydrogen bonding, $\pi-\pi$ stacking, and pore filling. The results showed that starch and $\gamma$-cyclodextrin polyurethane polymer adsorbents have excellent potential as adsorbent materials for the removal of phthalates from the contaminated water.
\end{abstract}

(c) 2014 Elsevier Ltd. All rights reserved.

\section{Introduction}

Phthalic acid esters (PAEs) or phthalates are a class of widely used industrial compounds known technically as dialkyl or alkyl aryl esters of 1,2-benzenedicarboxylic acid (phthalic acid). They have been widely used as important additive to give improved flexibility, durability, and workability in plastic industrial processes for the manufacture of different products including floor tiles, various types of household furnishing, food packaging, industrial and medical tubing, catheters, blood containers, and numerous other products (Llompart, Garcia-Jares, \& Landin, 2006, chap. 28). However, PAEs are reversibly bound to the polymer matrix of these plastic products (Bošnir et al., 2003; Llompart et al., 2006, chap. 28 ). Hence, they can migrate from the plastic to the external environment under certain conditions like high temperature, abrasion, leaching, etc., during the product life cycle of manufacture, use and disposal (Bauer \& Herrmann, 1997; Zeng et al., 2008). As

\footnotetext{
* Corresponding author. Tel.: +86 18701336534

E-mail addresses: guoqj@igsnrr.ac.cn, nonsokoli@gmail.com (Q.Guo).

1 Present address: Analytical/Environmental Chemistry Unit, Department of Chemistry, University of Ibadan, Nigeria.
}

a result of the large quantities produced, phthalate esters have become ubiquitous environmental pollutants. Considering their endocrine disrupting, carcinogenic, or xenoestrogenic properties (Tyler, Jobling, \& Sumpter, 1998), the presence of phthalates in water media has raised much public health concerns. In this vein, phthalates have been listed as one of the top priority pollutants by many environment authorities around the world. Thus, it is necessary to effectively and economically remove phthalates from the aquatic environment.

Review of past reports on remediation of phthalates polluted water has shown that adsorption technology has proved to be the most effective option (Julinová \& Slavík, 2012), and activated carbon has been the most common adsorbent applied for this purpose. However, high cost and difficult regeneration of spent activated carbon has left scientists with no other option than to develop alternative low cost adsorbents. Advances in polymer chemistry have led to the development of novel synthetically engineered adsorbent materials that utilize carbohydrate molecules such as starch, cyclodextrins, chitin, and chitosan for development of polymer adsorbents (Crini, 2005). Starch is essentially a condensation polymer of glucose molecules connected by $\alpha$-1,4-glycosidic linkages. Similar to starch, cyclodextrins are water soluble cyclic oligosaccharides composed of six, seven, or eight glucopyranose units 
linked by $\alpha$-1,4-glycosidic bonds; hereafter referred to as $\alpha-, \beta$ , and $\gamma$-cyclodextrin, respectively. Starch and cyclodextrin are of particular interest as adsorbent precursors because of their relative (cyclodextrin) abundance, specific structure, biodegradability, and chemical properties such as host-guest interaction (for cyclodextrin in particular) and high reactivity, resulting from the presence of chemically reactive hydroxyl groups in their molecular structure. The presence of the hydroxyl group makes chemical modification easy (Imam et al., 2012; Okoli, Guo, \& Adewuyi, 2014). Among various starch and cyclodextrin polymers, the cross-linked polymers have been the most investigated adsorbents due to their total biodegradability, biocompatibility, non-toxicity, cost effectiveness, stability on storage, as well as simple fabrication method (Kuniak \& Marchessault, 1972; Simkovic, Laszlo, \& Thompson 1996; Datskevich, 2009; Guo, Li, Liu, Yin, \& Li, 2009). Due to the host-guest interaction property of cyclodextrins, their polymers have been extensively studied for water remediation (Mohamed, Wilson, \& Headley, 2010). However, despite the fact that amongst $\alpha-, \beta-$, and $\gamma$-forms of cyclodextrin, $\gamma$-cyclodextrin has the largest molecular inclusion space $\left(174,262\right.$ and $427 \AA^{3}$ for $\alpha$-, $\beta$-, and $\gamma$-cyclodextrin, respectively) (Szejtli, 1998), limited studies on application of its polymers for water remediation have been reported.

It is against this backdrop that this study was designed to investigate the mechanism of adsorption of phthalates by $\gamma$-cyclodextrin and starch polymers, and explore the feasibility of applying these polymers as adsorbent for treatment of aqueous phthalates pollution.

\section{Materials and method}

\subsection{Materials}

Diethyl phthalate (DEP) was supplied by Tianjin Yongda Chemical Reagent Company Limited China, while Dimethyl phthalate (DMP) phthalate standard, 4,4-methylene diphenyl diisocyanate (MDI), and $\mathrm{N}, \mathrm{N}$-dimethyl formamide (DMF) were supplied by Aladin Chemistry Company Ltd, Shanghai, China. Sodium Azide $\left(\mathrm{NaN}_{3}\right)$ was supplied by Amresco, USA. Soluble starch (extracted from corn) was purchased from Guangfu Research Institute, Tianjin, while $\gamma$-cyclodextrin was supplied by Sinopharm, China. All the reagents are of analytical grade and were applied without further treatment. All solutions were prepared with ultra-pure water.

\subsection{Synthesis and characterization of adsorbents}

\subsubsection{Synthesis of adsorbents}

The $\gamma$-cyclodextrin polyurethane polymer (GPP) was prepared using cross-linking agent MDI and DMF as solvent by a one-step reticulation reaction. $8 \mathrm{~g}(6.17 \mathrm{mmol})$ aliquot of $\gamma$-cyclodextrin was dissolved in $40 \mathrm{ml}$ of DMF in a three-neck round bottom flask. Then $5.28 \mathrm{~g}(21.09 \mathrm{mmol})$ of MDI was gradually added while stirring at $70^{\circ} \mathrm{C}$ using a magnetic stirrer. The viscosity of the solution increased so strongly and rapidly that it could not be stirred after about $1 \mathrm{~h}$. At that point, acetone was added to stop the reaction, and the precipitated polymer was filtered off, washed copiously with distilled water, and further purified in a soxhlet unit using acetone for $12 \mathrm{~h}$. The polymer was finally lyophilized, and kept in a desiccator prior to use to prevent absorption of water from laboratory environment. The $\gamma$-cyclodextrin-starch polyurethane copolymer (GSP) was prepared by cross-linking a mixture of $4 \mathrm{~g} \gamma$-cyclodextrin ( $3.1 \mathrm{mmol})$ and $4 \mathrm{~g}$ starch with $5.28 \mathrm{~g}(21.09 \mathrm{mmol})$ of MDI, following a similar procedure of GPP adsorbent. The starch polyurethane polymer (SPP) was prepared in a similar procedure as described by Ozmen and Yilmaz (2007) and modified by Okoli et al. (2014) with
$8 \mathrm{~g}$ aliquot of starch and $5.28 \mathrm{~g}$ of MDI. The reaction schemes for the synthesis of the polymer adsorbents were as shown in Fig. S1.

\subsubsection{Characterization of adsorbents}

Fourier transform Infra-red (FT-IR) spectra of the synthesized adsorbent were obtained using Perkin Elmer Spectrum 1 FTIR spectrophotometer, using the scanning frequency of $4000-450 \mathrm{~cm}^{-1}$, to confirm the functional groups introduced as a result of the cross linking reaction. The specific surface area and porosity of the samples were determined via adsorption-desorption of nitrogen at $77 \mathrm{~K}$ using an ASAP 2020 Model (Micromeritics, USA) surface area and porosity analyzer. The morphologies of both $\gamma$-cyclodextrin and starch precursors, and their cross-linked polymer counterparts were examined at various magnifications using a scanning electron microscope (SEM) (Hitachi S4800 model, Japan).

\subsection{Adsorption studies}

\subsubsection{Adsorption set-up and procedure}

Batch adsorption method was adopted for this study using simulated DMP and DEP polluted water, prepared in synthetic ground water (deionized water containing $44 \mathrm{mg} / \mathrm{L} \mathrm{CaCl} 2 \cdot \mathrm{H}_{2} \mathrm{O}$ ) to mimic environmental water. $2000 \mathrm{mg} / \mathrm{L}$ phthalates (DMP and DEP) stock solutions were prepared in methanol, while the experimental solutions of desired concentrations were obtained by serial dilution using water. The methanol content was assumed to have no effect on solute equilibrium behavior due to its low concentration $(<0.1 \% \mathrm{v} / \mathrm{v})$ in the final experimental solution. Sodium azide $\left(\mathrm{Na}_{3} \mathrm{~N}\right)$ was added at a concentration of $200 \mathrm{mg} / \mathrm{L}$ to prevent phthalates biodegradation. Single solute phthalates solutions were applied for all the adsorption studies.

Batch adsorption experiments were carried out by adding aliquots of simulated phthalate polluted water samples into $100 \mathrm{ml}$ capacity glass stopped Erlenmeyer flasks containing desired dose of adsorbent. Aluminum foil was used to wrap the flasks to minimize possible losses by photochemical decomposition. The flasks were equilibrated in the dark by shaking at $170 \mathrm{rpm}$ using a horizontal shaker water bath. After equilibrium was attained, the phthalate solution was separated from the adsorbent by centrifugation at $3500 \mathrm{rpm}$ for $15 \mathrm{~min}$. Thereafter, equilibrium levels of phthalates were quantified using PerkinElmer series 200 (USA) High Performance Liquid Chromatography (HPLC) system with Brownlee Analytical (PerkinElmer, USA) reverse phase $C_{18}$ column $\left(150 \times 3.2 \mathrm{~mm}^{2}, 5 \mu \mathrm{m}, 110 \AA\right)$ equipped with Series $200 \mathrm{UV}$ Detectors. Phthalates were analyzed using isocratic elution with acetonitrile and water in the ratio of 70:30 and detected at UV absorbance of $254 \mathrm{~nm}$. All experiments were done in duplicates, and the mean values used for data analysis

The study of effect of contact time was done by agitating $50.0 \mathrm{mg}$ of adsorbent with $100 \mathrm{ml}$ aliquot of $8.0 \mathrm{mg} / \mathrm{L}$ of phthalates concentration while the residual phthalates concentration was monitored at $10,30,90,180,360,720$, and $1440 \mathrm{~min}$. The effect of $\mathrm{pH}$ was studied using solutions of different $\mathrm{pH}(3.72,5.10,6.80,8.40$, and 9.75), while the effect of ionic strength was studied using solutions of different ionic strength $\left(0,0.010 .05,0.10,0.25\right.$, and $0.5 \mathrm{M}$ of $\left.\mathrm{Na}^{+}\right)$that were prepared using $\mathrm{NaCl}$. The effect of adsorbent dose was studied by agitating $10,20,30,40,50,70$, and $100 \mathrm{mg}$ of polymer adsorbents with $50 \mathrm{ml}$ aliquot of $4.0 \mathrm{mg} / \mathrm{L}$ DEP solution. The effect of initial phthalate concentration was studied by varying the initial concentrations from 2.5, 5.0, 7.5, 10.0, 12.5, 15.0, 17.5, and $20.0 \mathrm{mg} \mathrm{L}^{-1}$ for both DMP and DEP. Study of the effect of temperature was carried out in a rate determination experiment at temperatures of 25 , 45 , and $60^{\circ} \mathrm{C}$ using $8.0 \mathrm{mg} \mathrm{L}^{-1}$ of DMP and DEP. All the adsorption experiments were performed in duplicates.

A blank study was done by adding a known mass of the adsorbent to a given volume of Milli- $Q$ water free of organic traces 
to check whether the adsorbent could release phthalates in the aqueous solution. For every experiment, adsorbent free phthalate solutions were subjected to the same experimental conditions to evaluate the loss of phthalates to factors, other than adsorption.

\subsubsection{Data treatment}

The equilibrium phthalates sorption capacity $q_{\mathrm{e}}(\mathrm{mg} / \mathrm{g})$, (quantity of phthalates adsorbed at equilibrium) was calculated using Eq. (1)

$q_{\mathrm{e}}=\frac{\left(C_{\mathrm{o}}-C_{\mathrm{e}}\right) V}{M}$

where $C_{\mathrm{o}}$ and $C_{\mathrm{e}}(\mathrm{mg} / \mathrm{L})$ are phthalates concentrations before and after adsorption, respectively; $V$ is the volume of the experimental solution (L); $M$ is the mass of sorbent $(\mathrm{g})$.

To gain insight into the kinetic mechanism, the data generated for effect of time were modeled into the pseudo first order (Lagergren, 1898), pseudo second order (Olu-Owolabi, Diagboya, \& Adebowale, 2014), Morris-Weber intra-particle diffusion and liquid film diffusion models (Qiu et al., 2009) as shown in Eqs. (2)-(5), respectively.

Pseudo first order (PFO) $\ln \left(q_{\mathrm{e}}-q_{\mathrm{t}}\right)=-k_{1} t+\ln q_{e}$

Pseudo second order (PSO) $\frac{t}{q_{\mathrm{t}}}=\frac{t}{q_{\mathrm{e}}}+\frac{1}{k_{2} q_{\mathrm{e}}^{2}}$

where, $q_{\mathrm{e}}$ and $q_{\mathrm{t}}$ are the amounts of phthalates sorbed $(\mathrm{mg} / \mathrm{g})$ at equilibrium and at any time, $t$ respectively, $k_{1}(1 / \mathrm{min})$ is the pseudofirst order rate constant, and $k_{2}(\mathrm{~g} / \mathrm{mg} / \mathrm{min})$ is the pseudo-second order rate constant. The initial rate constant $h$, is calculated as $h=k_{2} q_{\mathrm{e}}{ }^{2}$. The values of $k_{2}, h$, and $q_{\mathrm{e}}$ for pseudo second order model were evaluated from the linear plots of $t / q_{\mathrm{t}} \mathrm{vs}$. $t$, while the values of $k_{1}$, and $q_{\mathrm{e}}$ for pseudo first order were evaluated from the linear plots of $\ln \left(q_{\mathrm{e}}-q_{\mathrm{t}}\right)$ vs. $t$.

Morris - Weber intra - particle diffusion $q_{\mathrm{t}}=k_{\mathrm{id}} t^{1 / 2}+C_{\mathrm{i}}$

Liquid film diffusion $\ln \left(1-\frac{q_{\mathrm{t}}}{q_{\mathrm{e}}}\right)=-R^{i} t$

where $k_{\text {id }}$ is the diffusion rate parameter for intraparticle diffusion model, $C_{\mathrm{i}}$ is a constant related to adsorption on the surfaces of the pores, $q_{\mathrm{t}} / q_{\mathrm{e}}$ is the fractional attainment of the equilibrium, and $R^{i}$ is the diffusion rate parameter for film diffusion model.

To obtain some insights into the surface properties and degree of affinity of the sorbent, sorption data has been fitted to the Langmuir, Freundlich, BET, and Tempkin equilibrium isotherms using the linear forms of these models (Febrianto et al., 2009) as shown in Eqs. (6)-(9).

Langmuir equation $\frac{1}{q_{\mathrm{e}}}=\frac{1}{q_{\max }}+\frac{1}{q_{\max } K_{\mathrm{L}}}\left(\frac{1}{C_{\mathrm{e}}}\right)$

Freundlich isotherm $\log q_{\mathrm{e}}=\log K_{\mathrm{f}}+\frac{1}{n} \log C_{\mathrm{e}}$

where $q_{\max }(\mathrm{mg} / \mathrm{g})$ and $q_{\mathrm{e}}$ is the maximum adsorption and amount of solute adsorbed per unit weight of adsorbent $(\mathrm{mg} / \mathrm{g})$, respectively. $C_{\mathrm{e}}$ is same as above; $K_{\mathrm{L}}, K_{\mathrm{f}}$, and $n$ are isotherm constants obtained from the slopes and intercepts.

BET isotherm $\frac{C_{\mathrm{e}}}{\left(C_{\mathrm{s}}-C_{\mathrm{e}}\right) q_{\mathrm{e}}}=\left(\frac{K_{\mathrm{B}}-1}{K_{\mathrm{B}} Q^{0}}\right) \frac{C_{\mathrm{e}}}{C_{\mathrm{s}}}+\frac{1}{K_{\mathrm{B}} Q^{0}}$

where $C_{\mathrm{s}}$ is the saturation concentration (solubility limit) of the solute in $\mathrm{mg} / \mathrm{L}, K_{\mathrm{B}}$ is a constant related to the energy of interaction with the surface and the subsequent layers of adsorbates and $Q^{\circ}$ is the amount of solute adsorbed per unit weight of adsorbent in forming a complete monolayer on the surface.

$$
\begin{array}{cc}
\text { Tempkin isotherm } & q_{\mathrm{e}}=B \ln A+B \ln C_{\mathrm{e}} \\
& \text { while } B=\frac{R T}{b}
\end{array}
$$

where $R, T$ and $b$ are the gas constant, the absolute temperature in Kelvin, and the constant related to the heat of adsorption, respectively. $C_{\mathrm{e}}$ and $q_{\mathrm{e}}$ are as defined earlier, and $A$ is the Temkin isotherm constant.

The adsorption thermodynamic parameters; enthalpy $\left(\Delta H^{\circ}\right)$, entropy $\left(\Delta S^{\circ}\right)$, Gibbs free energy $\left(\Delta G^{\circ}\right)$ and equilibrium constant $(K c)$, were evaluated for the experimental systems using van't Hoff and Gibbs-Helmholtz equations (Olu-Owolabi, Diagboya, \& Ebaddan, 2012) as shown in Eqs. (10)-(12).

$$
\begin{aligned}
\ln K_{\mathrm{c}} & =\frac{q_{\mathrm{e}}}{C_{\mathrm{e}}} \\
\ln K_{\mathrm{c}} & =-\frac{\Delta H^{\circ}}{R T}+\frac{\Delta S^{\circ}}{R} \\
\Delta G^{\circ} & =\Delta H^{\circ}-T \Delta S
\end{aligned}
$$

\section{Result and discussion}

\subsection{Characterization of adsorbents}

Fig. 1 shows the FTIR spectra of pristine starch, $\gamma$-cyclodextrin, GPP, GSP, and SPP. The non-existence of $-\mathrm{N}=\mathrm{C}=\mathrm{O}$ band $\left(2285-2250 \mathrm{~cm}^{-1}\right)$ in the spectra of the three polymers (GPP, GSP, and SPP) showed that the two isocyanate groups of the MDI molecule were involved in the cross-linking process. The spectra for all the polymers showed sharp peak around $1510 \mathrm{~cm}^{-1}$ signifying the $\mathrm{N}-\mathrm{H}$ deformation of secondary amides (amide-II band), and $1649 \mathrm{~cm}^{-1}$ signifying $C=0$ stretching vibration in secondary amides (amide-I band) as labeled in Fig. 1. These peaks are in consonance with the formation of amide groups $(-\mathrm{CONH}-)$, as shown in the reaction scheme for the synthesis of the polymer (Fig. S1 of the Supplementary material). The peak at $1595 \mathrm{~cm}^{-1}$ signifying ring stretching vibration of benzene ring in aromatic compound, aromatic $\mathrm{C}-\mathrm{H}$ (3029 and $3027 \mathrm{~cm}^{-1}$ for GPP and GSP, respectively), and C - C (1410 and $1406 \mathrm{~cm}^{-1}$ for GPP and GSP, respectively) confirmed successful cross-linking with MDI molecule and successive incorporation of aromatic group into the chemical structural matrix of these adsorbents. Also, the presence of stretching vibration of $\mathrm{C}-\mathrm{H}$ bonds of glucose units $\left(2905 \mathrm{~cm}^{-1}\right), \mathrm{C}-\mathrm{O}\left(1320-1000 \mathrm{~cm}^{-1}\right)$ and $\mathrm{O}-\mathrm{H}\left(3500-3200 \mathrm{~cm}^{-1}\right)$ stretching vibrations of polymeric compounds especially polysaccharides, in addition to the characteristic broad peaks of anomeric C-H ring deformations ( 635 and $645 \mathrm{~cm}^{-1}$ for GPP and GSP, respectively), showed that both the starch and cyclodextrin structural backbones were retained in the cross-linked polymer.

Table 1 showed the results of surface and pore analysis of the polymer adsorbents. The values of BET specific surface area of the synthesized adsorbents were higher than the established values of the adsorbent precursor materials $\left(0.980 \mathrm{~m}^{2} / \mathrm{g}\right.$ and $0.712 \mathrm{~m}^{2} / \mathrm{g}$ for starch and $\gamma$-cyclodextrin, respectively). Hence, it can be concluded that cross-linking process led to improvement in the surface and porosity characteristics of the cross-linked polymer adsorbents. This is in tandem with the established principle that cross-linking process increases the network of the starch polymer, thus increasing the surface area (Crini, 2005). Analysis of the BET total surface area in relation to the adsorption surface area of pores is necessary for understanding the adsorption mechanism and behavior of adsorbents, since the information reveals the extent of adsorption that takes place on the exterior, as well as the interior surfaces of 

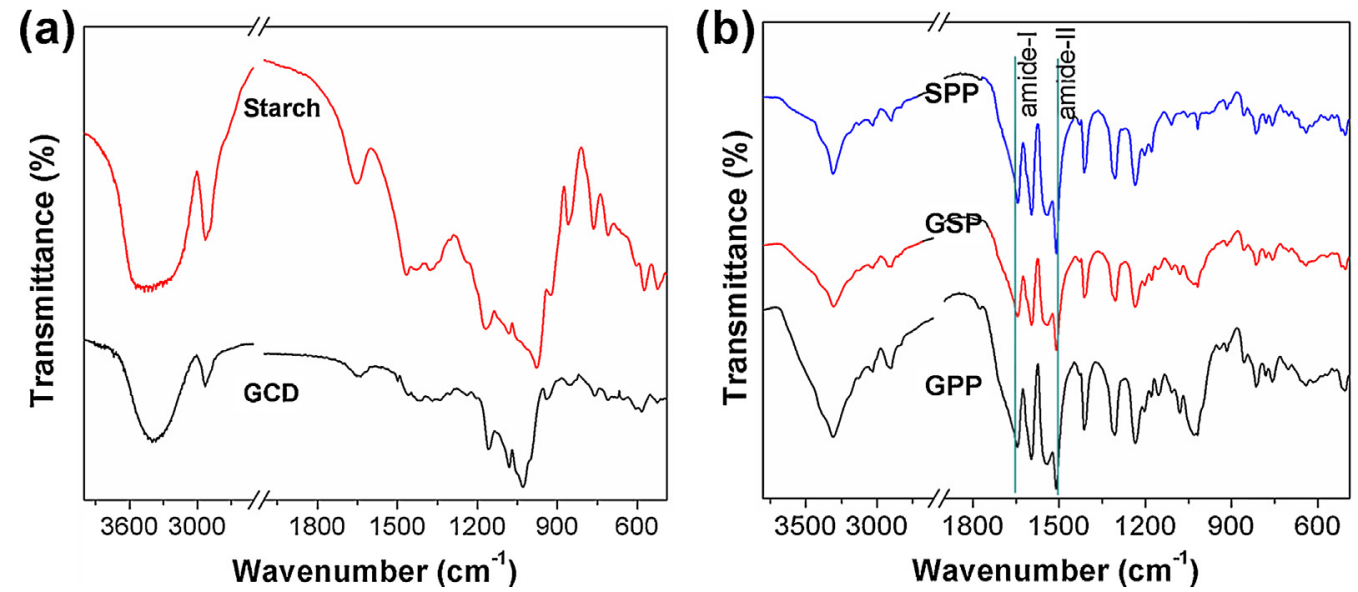

Fig. 1. FTIR spectra of (a) adsorbent precursors (starch and $\gamma$-cyclodextrin) and (b) cross-linked polymer adsorbents (GPP, GSP, and SPP).
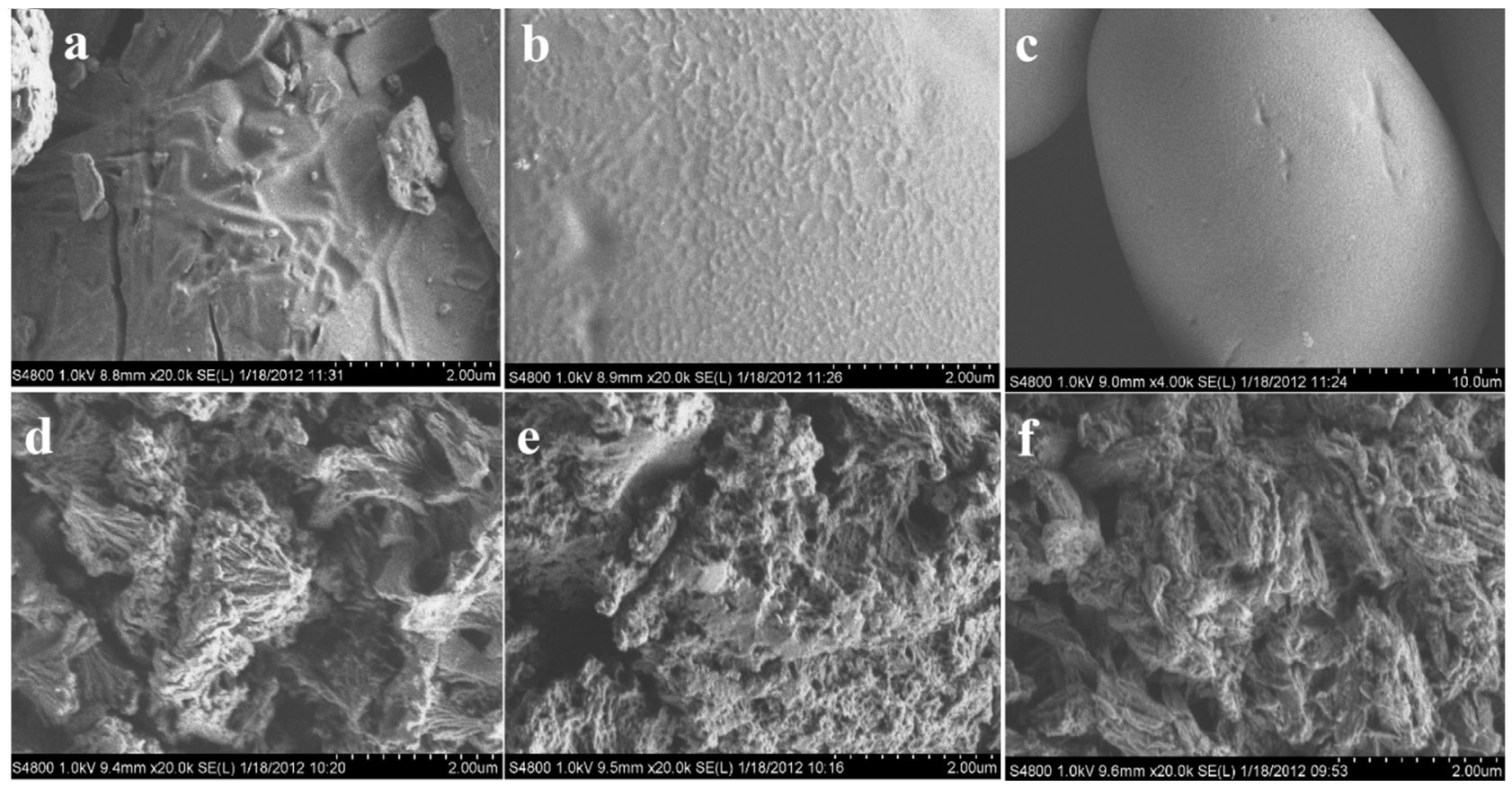

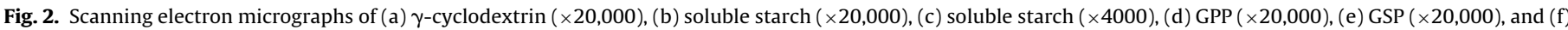
SPP $(\times 20,000)$

the adsorbents. In this regards, the pore surface/total surface area ratio of the adsorbents shows that though SPP might have had the highest total surface area, higher percentage of the likely adsorption process is expected to take place in the internal pore surfaces. Hence, SPP is predicted to have the slowest adsorption rate while GPP and GSP adsorbents are expected to have similar rates. Also, the study revealed that the average pore widths and pore volumes for most of the adsorbents are large enough to allow diffusion of adsorbate molecules into the internal surfaces of the adsorbents.

The SEM images (Fig. 2) revealed that the surface of the $\gamma$ cyclodextrin (Fig. 2a) and starch (Fig. 2b and c) precursors were relatively smooth, while the surface of the cross linked polymer counterparts (GPP-Fig. 2d, GSP-Fig. 2e, and SPP-Fig. 2f) had noticeable pores, thus corroborating higher BET surface areas

Table 1

Summary of BET surface and pore analysis of the polymer adsorbents.

\begin{tabular}{|c|c|c|c|}
\hline Adsorbent & GPP & GSP & SSP \\
\hline BET surface area $\left(\mathrm{m}^{2} / \mathrm{g}\right)$ & 15.2145 & 7.6178 & 40.3858 \\
\hline $\mathrm{BJH}$ adsorption cumulative surface area of pores $\left(\mathrm{m}^{2} / \mathrm{g}\right)^{\mathrm{a}}$ & 10.6690 & 5.3530 & 40.0851 \\
\hline Pore surface/Total surface area & 0.7012 & 0.7026 & 0.9925 \\
\hline t-Plot external surface area $\left(\mathrm{m}^{2} / \mathrm{g}\right)$ & 21.4654 & 8.8089 & 58.6268 \\
\hline BJH adsorption pore volume ${ }^{a}$ & 0.0623 & 0.0478 & 0.1881 \\
\hline BET adsorption average pore width $(4 \mathrm{~V} / \mathrm{A})(\AA)$ & 98.0814 & 139.7694 & 128.5709 \\
\hline BJH adsorption average pore width $(4 \mathrm{~V} / \mathrm{A})(\AA)^{\mathrm{a}}$ & 233.7440 & 356.8720 & 187.7271 \\
\hline
\end{tabular}

\footnotetext{
a For pores between 17,000 and $3000,000 \AA$ width .
} 

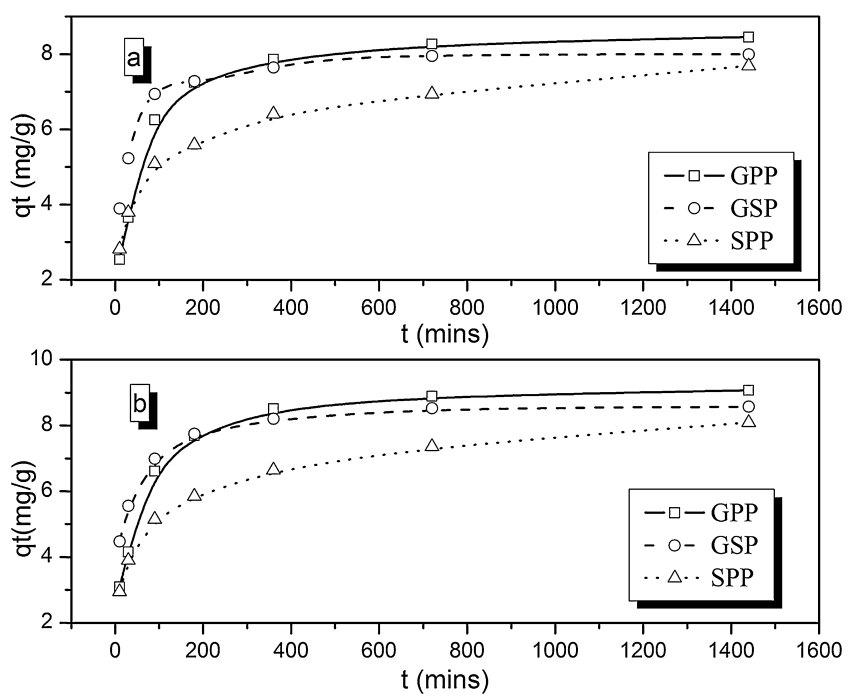

Fig. 3. Effect of contact time on (a) DMP and (b) DEP adsorption onto the polymer adsorbents.

observed for the cross-linked adsorbents. The SEM images revealed that the particles of $\gamma$-cyclodextrin and starch were globular in shape and of different sizes while the cross linked polymer counterparts were agglomerates of irregular shapes and sizes, indicating the formation of bulkier polymer units emanating from the cross linking process. These changes in micro-physical structure also confirmed the success of the adsorbent synthesis.

\subsection{Adsorption study}

\subsubsection{Effect of contact time and adsorption dynamics}

Considering the fact that industrial wastewaters cannot be held in treatment plants for long periods, it is imperative that maximum sorption occurs in short time. In this regards, there is need to establish the phthalates adsorption-time relationship for investigating various process conditions for the designing of water clean-up procedures.

The adsorption rate curves (Fig. 3) showed that adsorptions for GPP and GSP were rapid in the first $90 \mathrm{~min}$, with $72.9 \%$ $(6.7 \mathrm{mg} / \mathrm{g})$ and $81.6 \%(7.0 \mathrm{mg} / \mathrm{g})$, respectively, of the total DEP adsorption occurring within this time frame. As time elapsed, the rate decreased significantly until after $400 \mathrm{~min}$ when the percentage of DEP adsorption increased to $98.0 \%(8.9 \mathrm{mg} / \mathrm{g})$ and $99.9 \%$ $(8.5 \mathrm{mg} / \mathrm{g})$ for GPP and GSP, respectively. This trend of adsorption may be explained thus: the initial rapid increase in DEP uptake is due to the fact that a large number of vacant surface sites on the cross-linked adsorbent are available for adsorption during the initial stage and since these have very high affinity for DEP, they are filled rapidly. As time elapsed, the number of vacant adsorption sites progressively decreased resulting to the observed slow adsorption rate.

The rate curve for the adsorption of SPP adsorbent was somewhat different from that of GPP and GSP counterparts (Fig. 3). The curve showed that DEP adsorption for SPP was less rapid than those of GPP and GSP adsorbents, with less than $65 \%(5.2 \mathrm{mg} / \mathrm{g})$ of the total DEP adsorption occurring in the first $90 \mathrm{~min}$, and thereafter increased to $90.9 \%(7.4 \mathrm{mg} / \mathrm{g})$ in $720 \mathrm{~min}$. Unlike that of GPP and GSP adsorbents, significant level of adsorption still occurred after $720 \mathrm{~min}$. Hence, the time required to attain a rapid equilibrium is $720 \mathrm{~min}$. However, a contact time of $1440 \mathrm{~min}$ has been employed for every other study because of this possibility of further adsorption. This observation can be explained on the basis of the pore and surface area make-up of the adsorbents. The result of pore and surface area analysis (Table 1) of the adsorbents showed that SPP had the highest value for ratio of surface area of pores to total surface area (value of 0.9925). This implies that most of the adsorption sites for this adsorbent are located on the pore surfaces; hence over one half of SPP adsorption occurred in the internal pore surfaces. Therefore, more time was required to reach these sites than those on the external surfaces. The rate curves of DMP adsorption on the studied adsorbents also followed similar trends observed on DEP adsorption. Hence, the same explanation for DEP adsorption will suffice for these similar observations.

It has been shown that adsorption removal of adsorbate molecules from aqueous phase system proceed via three successive steps viz: (a) transport of molecules from the bulk of the solution to the exterior surface of porous adsorbent particles through a boundary layer (liquid film or external diffusion); (b) diffusion of the molecules through the interior pores of the adsorbent (intraparticle or internal diffusion); and (c) adsorption of molecules onto the active sites on the interior surface (Qiu et al., 2009). All the three steps could contribute to the overall adsorption kinetics, while the third step also controls the specific adsorption capacity and intensity. More often, one of the 3 steps offers the greatest resistance and is thus referred to as the rate limiting step of the whole sorption process. In practice, unless chemical modifications occur during the sorption, the third stage is assumed to be too fast to contribute significantly to the overall sorption rate. It is thus generally understood that slow sorption kinetics are caused by rate-limiting diffusive mass transfer. It is based on these concepts that the four kinetics models: two reactive (pseudo-first order and pseudo-second order) and two diffusion based models (intra-particle and liquid film diffusion models) were used to test the mechanism that controlled the sorption process.

In this regards, the kinetic parameters (Fig. S2 and Table 2 showing the plots and comparative fits, respectively) of the various models indicate that the adsorption of phthalate molecules onto the active sites on the surfaces of the adsorbents fit the pseudosecond-order kinetics better than for pseudo-first-order kinetics because the $r^{2}$ values as well as the experimental $q_{\mathrm{e}}$ values of the pseudo-second-order are better correlated than that of pseudo first order. Liquid film and intra-particle diffusion models gave further insight into the controlling diffusion mechanism involved in the adsorption process. The fits of intra-particle diffusion curves indicate that diffusion of the phthalate molecules through the interior pores of the adsorbent had significant contribution to the overall adsorption kinetics. However, the fact that the curves did not pass through the origin $\left(C_{\mathrm{id}} \neq 0\right)$ reveals that an initial boundary layer resistance existed, and equally indicated that the adsorption process was not solely dominated by pore diffusion. Table 2 showed that the fits of liquid film diffusion model were better than that of intra-particle diffusion model. This indicated that transport of phthalate molecules from the bulk of the solution to the exterior surface of cross-linked adsorbents is the rate controlling step.

\subsubsection{Effect of adsorbent dose}

Fig. 4 shows the plots of $q_{\mathrm{e}}$ and \% removal against varying adsorbent dose (10-100 mg). It was observed that increasing the adsorbent dose in the studied range increased the \% removal of DEP from 33.0-77.7\%, 31.0-92.3\%, and 30.7-93.4\% for GPP, GSP, and SPP, respectively. On the other hand, the equilibrium adsorption capacity $q_{\mathrm{e}}$ per unit mass of the studied adsorbents was found to decrease $6.4-1.5,6.0-1.8$, and $5.9-1.8 \mathrm{mg} / \mathrm{g}$ for GPP, GSP, and SPP, respectively, with increasing adsorbent dose. Similar reports are found in literature (Chan, Lau, Ang, Wu, \& Wong, 2004). It was apparent that by increasing the amount of cross-linked adsorbent, available sorption sites for sorbent-solute interaction is increased due to increased available active surface adsorption sites, hence, leading to the noticed increased percentage phthalate removal from the 
Table 2

Kinetic Parameters for the adsorption of DMP and DEP onto GPP, GSP, and SPP polymer adsorbents.

\begin{tabular}{|c|c|c|c|c|c|c|}
\hline Kinetic model & GPP/DMP & GPP/DEP & GSP/DMP & GSP/DEP & SPP/DMP & SPP/DEP \\
\hline$q_{\mathrm{e}}$ Expt & 8.4574 & 9.0720 & 7.9959 & 8.5726 & 7.688 & 8.0944 \\
\hline \multicolumn{7}{|c|}{ Psuedo first order } \\
\hline$q_{e}$ calc & 3.2160 & 3.3601 & 1.8288 & 2.0924 & 3.9743 & 4.2691 \\
\hline$K_{1}$ & 0.0023 & 0.0022 & 0.0025 & 0.0024 & 0.0024 & 0.0022 \\
\hline$r^{2}$ & 0.7941 & 0.7965 & 0.7629 & 0.7726 & 0.9749 & 0.9812 \\
\hline \multicolumn{7}{|c|}{ Psuedo second order } \\
\hline$q_{e}$ calc & 8.6535 & 9.2730 & 8.0854 & 8.6798 & 7.8162 & 8.2549 \\
\hline$K_{2}$ & 0.0034 & 0.0033 & 0.0078 & 0.0066 & 0.0023 & 0.0022 \\
\hline$h$ & 0.2546 & 0.2894 & 0.5099 & 0.5028 & 0.1507 & 0.1514 \\
\hline$r^{2}$ & 0.9999 & 0.9998 & 0.9999 & 0.9999 & 0.9963 & 0.9964 \\
\hline \multicolumn{7}{|c|}{ Intra-particle diffusion } \\
\hline$K_{i d}$ & 0.1581 & 0.1629 & 0.1001 & 0.1073 & 0.1302 & 0.1404 \\
\hline$C_{i d}$ & 3.7239 & 4.1811 & 5.0568 & 5.3875 & 3.3314 & 3.3990 \\
\hline$r^{2}$ & 0.6454 & 0.6718 & 0.5695 & 0.6500 & 0.8543 & 0.8739 \\
\hline \multicolumn{7}{|c|}{ Liquid film diffusion } \\
\hline$K_{f d}$ & 0.0023 & 0.0022 & 0.0025 & 0.0023 & 0.0024 & 0.0023 \\
\hline$r^{2}$ & 0.7940 & 0.7965 & 0.7629 & 0.7726 & 0.9749 & 0.98115 \\
\hline
\end{tabular}

aliquot solution. Olu-Owolabi et al. (2012) have attributed the $q_{\mathrm{e}}$ decrease with increasing mass to the decreasing total surface area of the adsorbent and an increase in diffusion path length due to aggregation of adsorbent particles, and as the weight of the crosslinked adsorbents increased, the aggregation becomes increasingly significant.

\subsubsection{Effect of $\mathrm{pH}$ and salinity ( $\mathrm{Na}^{+}$ions) on phthalates adsorption}

The result of study for effect of $\mathrm{pH}$ as shown in Table 3 indicated that changes in the aqueous $\mathrm{pH}$ did not significantly affect adsorption of diethyl phthalate onto GPP and GSP polymer adsorbents. The observation can be attributed to the neutrality of the adsorbent surfaces, as previous studies on adsorption of phthalates had established that the effect of $\mathrm{pH}$ on adsorption of phthalates often emanates from the $\mathrm{pH}$ effect on charge properties of the adsorbent surface, rather than the phthalate molecules (Staples, Peterson, Parkerton, \& Adams, 1997; Chen \& Chung, 2006). Since none of the adsorbents under study has charged surfaces, $\mathrm{pH}$ was therefore supposed to have minimal effect on their adsorption performance for phthalates, as observed in this study. However, the adsorption capacity of SPP adsorbent was enhanced at relatively high aqueous $\mathrm{pH}$ of the solution. This observation can be attributed to the fact that at relatively high $\mathrm{pH}$, there will be de-protonation of

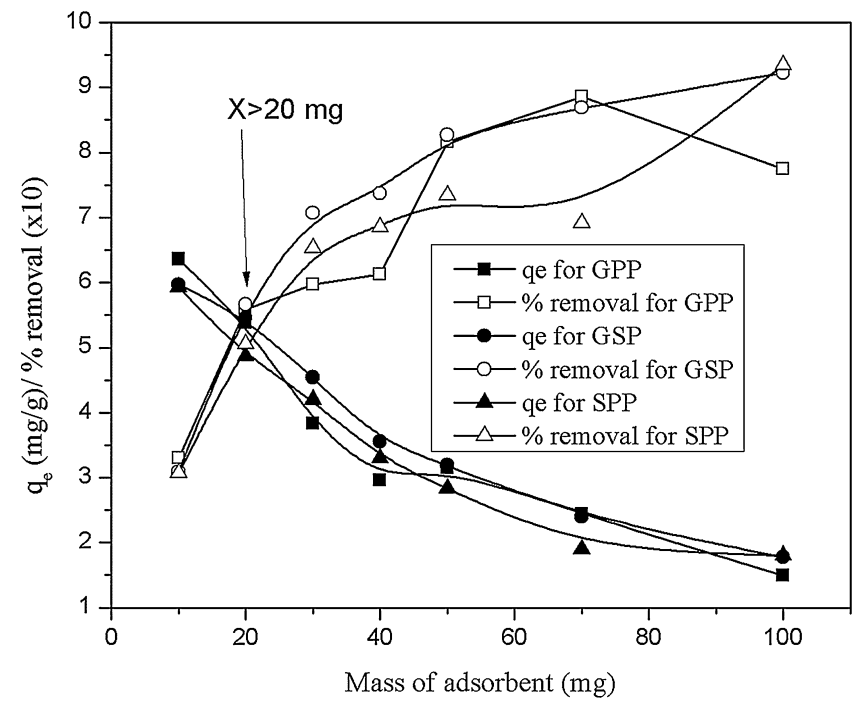

Fig. 4. Effect of adsorbent dose on adsorption capacity and \% removal. the surface $\mathrm{OH}$ groups of the adsorbents, thus leaving residual negative charges on the adsorbent surfaces. The aromatic moieties of the phthalate molecules interact more with these charged surfaces, and thereby enhance adsorption. However, the extent of manifestation of this effect is subject to the polarity of the surfaces. Considering the structure of cross-linked cyclodextrin and starch polymers (Fig. S1), it is obvious that this effect will manifest more on starch polymer (SPP), since starch is more polar than cyclodextrin. Hence, this is the reason behind the observed difference in behavior of the studied adsorbents towards variation in $\mathrm{pH}$.

The results of the effect of $\mathrm{Na}^{+}$(representative of salinity) on phthalate adsorption (Table 3 ) showed that increases in salinity ( $\mathrm{NaCl}$ concentration) did result to corresponding increment in adsorption capacity of all the studied adsorbents. This can be attributed to the reductive effect of $\mathrm{Na}^{+}$on the solubility of phthalates. Hence, the reduction in the solubility of phthalates is what actually enhanced the adsorption process. This result suggests that GPP, GSP, and SPP adsorbents could be used as adsorbents in freshwater and seawater aquaculture systems.

\subsubsection{Effect of initial phthalate concentration and isotherm studies}

Equilibrium phthalate adsorption data (Fig. S3) showed that increasing the phthalate aqueous concentration resulted in higher adsorption of phthalates from solution. The increase in sorption with increase in solution concentration of phthalates was attributed to the fact that when the polymer adsorbents' external surface film and internal pores transports of phthalates are

Table 3

Effect of $\mathrm{pH}$ and salinity on the adsorption of DEP onto cross-linked polymer adsorbents.

\begin{tabular}{llll}
\hline Parameter & \multicolumn{2}{l}{ Adsorption capacity $\left(q_{\mathrm{e}}\right)(\mathrm{mg} / \mathrm{g})$} & \\
\cline { 2 - 4 } & GPP & GSP & SPP \\
\hline pH values & & 6.22 & 6.16 \\
3.72 & 6.44 & 6.36 & 6.27 \\
5.10 & 6.58 & 6.14 & 5.75 \\
6.80 & 6.36 & 6.25 & 6.23 \\
8.40 & 6.45 & 6.37 & 6.58 \\
9.75 & 6.47 & & \\
Salinity $\left[\mathrm{Na}^{+}\right]$ & & 6.07 & 5.94 \\
0.00 & 5.94 & 6.05 & 5.96 \\
0.01 & 5.96 & 6.12 & 6.08 \\
0.05 & 6.08 & 6.17 & 6.08 \\
0.10 & 6.08 & 6.05 & 6.10 \\
0.25 & 6.10 & 6.41 & 6.43 \\
0.50 & 6.44 & & \\
\hline
\end{tabular}



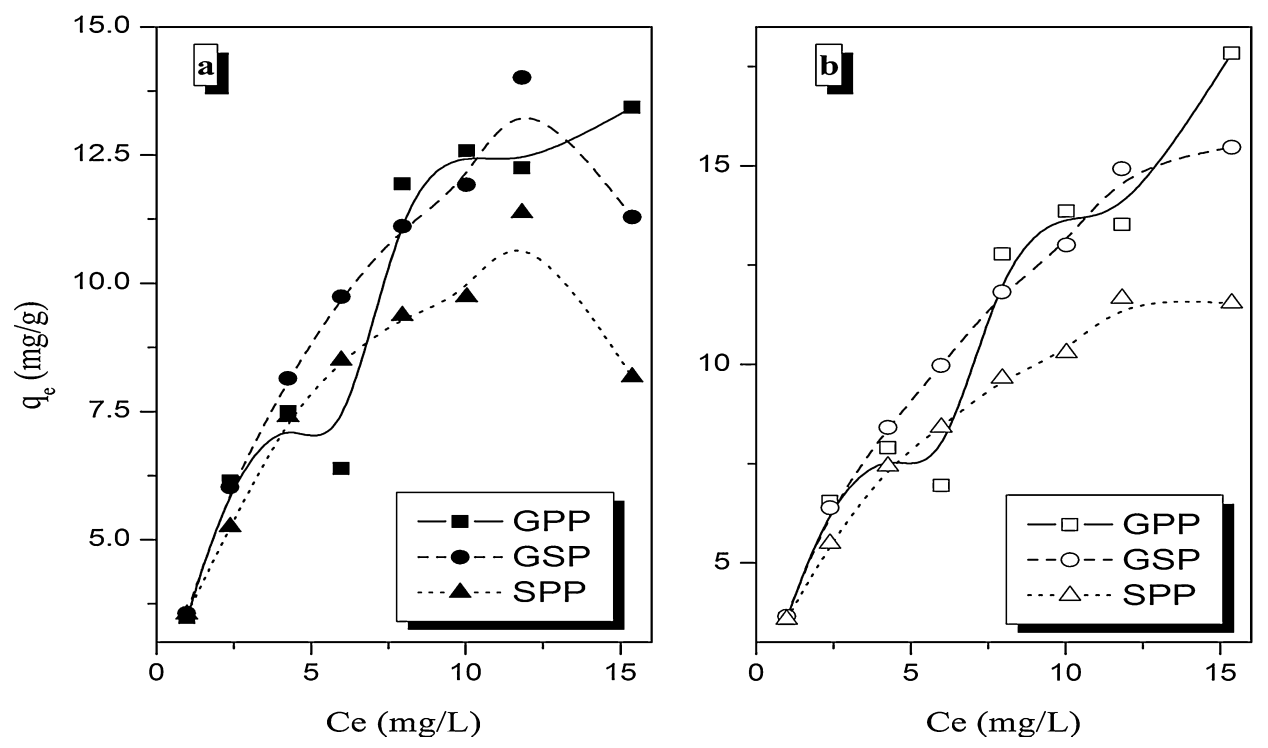

Fig. 5. Experimental isotherm plots of (a) DMP and (b) DEP adsorption on the polyurethane polymer adsorbents (GPP, GSP, and SPP).

equal, the trans-boundary movement of phthalates will not be significantly permissible; however, increasing concentration will re-initiate the trans-boundary movement of phthalates and sorption will be concentration dependent.

The experimental isotherm plots for the adsorption of phthalates onto the cross-linked starch and cyclodextrin polymer adsorbents were as shown in Fig. 5. The plots showed that the equilibrium sorption curves for the studied adsorption mainly followed the L-type isotherm profiles; adsorption of DMP and DEP by GSP and SPP exhibited more of L-type isotherm, while that of GPP adsorbent exhibited the borderline of L-type and S-type isotherm profiles, with the L-type being the dominant profile. Considering the conditions for exhibition of this isotherm profile as explained by Giles, Macewan, Nakhwa, and Smith (1960), it can be concluded that there was no competition from the aqueous phase for the adsorption sites of the cross-linked adsorbents.

The correlation coefficients $\left(r^{2}\right)$ and the $q_{\mathrm{e}}$ values of the adsorption isotherm data (Table 4) showed that the Langmuir adsorption isotherm model could be used to describe the phthalate adsorption data. This is an indication that the adsorption of phthalates on these adsorbents were on adsorption sites with similar energy. The $r^{2}$ values of the Freundlich and BET adsorption isotherm model (Table 4) and nonlinear isotherm plots (Figs. S4 and S5) showed that these models could describe the adsorption data. Though traditionally, the Freundlich and BET isotherm implies multi-layer adsorption on heterogeneous surfaces, this is partly true of the adsorption on these adsorbents. As stated earlier, the surface and porosity analysis showed that the adsorbents are porous and hence, adsorption of phthalates occurs on both the external surfaces of the adsorbents, as well as the internal pores. However, the fitting of the data to the Langmuir isotherm implied that the external and the internal surfaces have similar adsorption energy. Brunauer, Emmett, and Teller (1938) have shown that several Langmuir type adsorptions on a single adsorbent can be described by the Freundlich and BET isotherm models. Hence, the fitting of the Freundlich and BET isotherms as observed in this study can be explained in a similar vein.

Also, the fact that both Langmuir and Freundlich adsorption isotherm models gave good fittings of the adsorption data indicates that the interactions that took place between the adsorbents and phthalate molecules are weak interactions like hydrogen bonding dipole, and $\pi-\pi$ interactions. The existence of the weak interactions can be explained on the basis of chemical structural properties of both the polyurethane polymer adsorbents and the phthalate molecules as elucidated in Fig. 6. The presence of phenyl ring on the adsorbents is favourable to increase the adsorption capacity and affinity of the aromatic ring on DMP and DEP molecules by $\pi-\pi$ interaction. On the existence of hydrogen bonding, the hydrogen atom of the hydroxyl group on the polyurethane polymer chain

Table 4

Isotherm model parameters for DMP and DEP adsorption using the studied adsorbents.

\begin{tabular}{|c|c|c|c|c|c|c|c|}
\hline \multirow[t]{2}{*}{ Isotherm models } & \multirow[t]{2}{*}{ Parameters } & \multicolumn{2}{|l|}{ GPP } & \multicolumn{2}{|l|}{ GSP } & \multicolumn{2}{|l|}{ SSP } \\
\hline & & DMP & DEP & DMP & DEP & DMP & DEP \\
\hline & $\operatorname{Exp} q_{\mathrm{e}}$ & 13.4278 & 17.8332 & 11.2869 & 15.4680 & 8.1765 & 11.54025 \\
\hline \multirow[t]{3}{*}{ Langmuir } & $r^{2}$ & 0.8767 & 0.8824 & 0.9858 & 0.9878 & 0.9364 & 0.9631 \\
\hline & $q_{\max }$ & 13.6463 & 15.8200 & 15.1012 & 17.3800 & 10.8944 & 12.1374 \\
\hline & $K_{\mathrm{L}}$ & 0.3329 & 0.3025 & 0.3073 & 0.2767 & 0.4691 & 0.4131 \\
\hline \multirow[t]{3}{*}{ Freundlich } & $r^{2}$ & 0.8281 & 0.8442 & 0.9292 & 0.9929 & 0.8385 & 0.9876 \\
\hline & $K_{\mathrm{f}}$ & 3.6045 & 3.7300 & 3.9092 & 3.9400 & 3.8232 & 1.7600 \\
\hline & $n$ & 2.0400 & 1.8400 & 2.1100 & 1.8500 & 2.6500 & 2.2900 \\
\hline \multirow[t]{3}{*}{ BET } & $r^{2}$ & 0.6696 & 0.5211 & 0.9352 & 0.9779 & 0.9018 & 0.9857 \\
\hline & $Q^{\circ}$ & 200.2659 & 22.3625 & 358.5304 & 20.0868 & 719.0829 & 13.7770 \\
\hline & $K_{\mathrm{B}}$ & 17.1055 & 81.9345 & 14.7149 & 115.4324 & 10.1574 & 163.5039 \\
\hline \multirow[t]{3}{*}{ Tempkin } & $r^{2}$ & 0.7586 & 0.7391 & 0.8943 & 0.9632 & 0.7728 & 0.9702 \\
\hline & $A$ & 2.1316 & 1.7230 & 2.6117 & 1.9700 & 4.4185 & 2.9000 \\
\hline & $b$ & 670.2400 & 530.3800 & 698.0700 & 554.7800 & 1023.5000 & 810.7700 \\
\hline
\end{tabular}




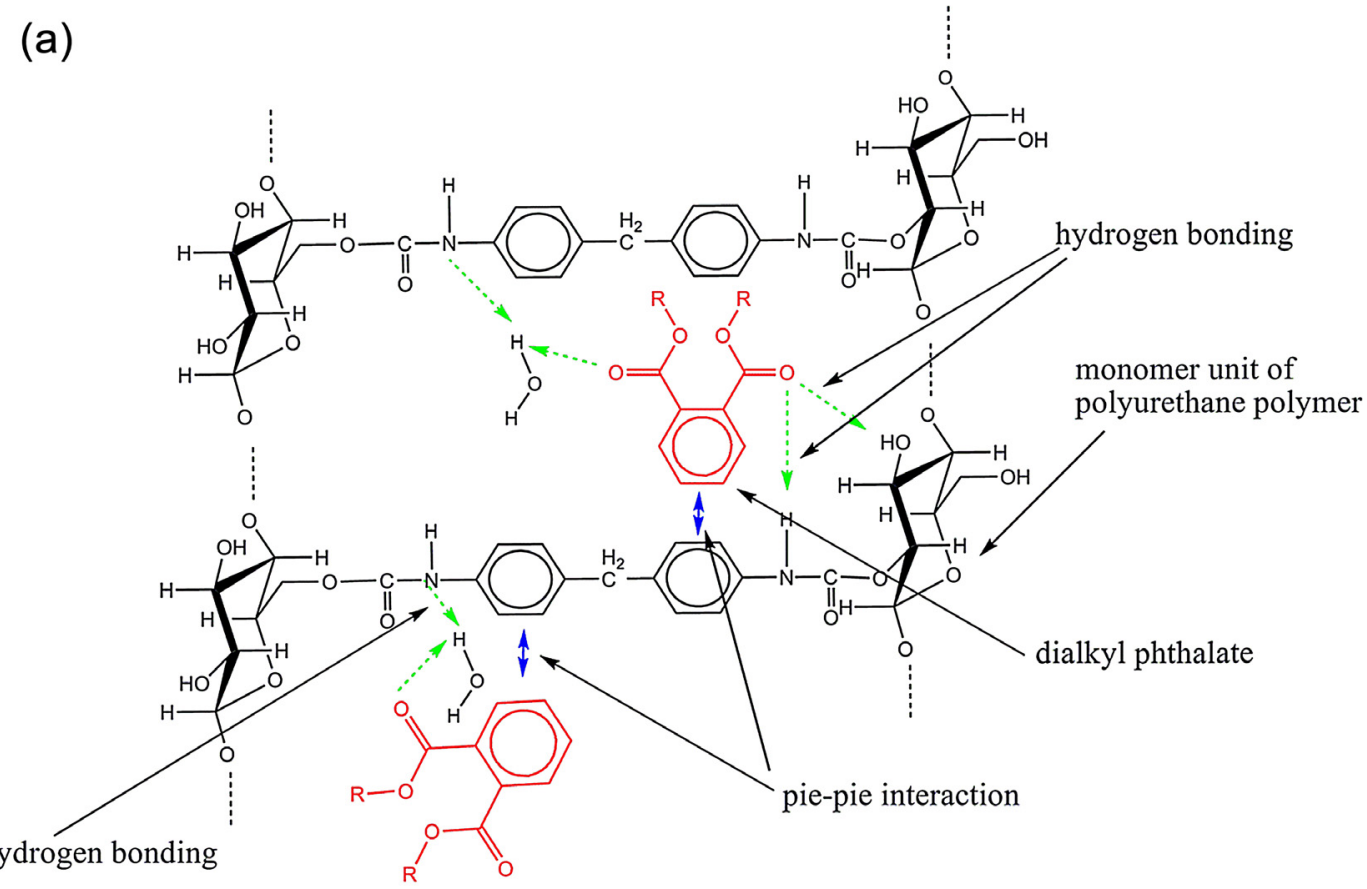

(b)

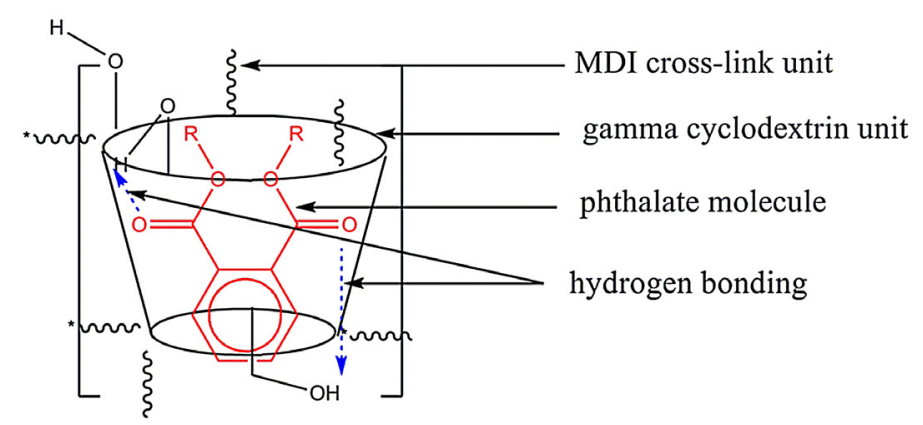

Fig. 6. Schematics of the adsorption interaction of phthalates with the polyurethane polymer on (a) interfacial and (b) inclusion spaces of the adsorbents.

of the cross-linked adsorbents can directly act as hydrogen bonding acceptor and form hydrogen bonding with the oxygen atom of the ester group of DMP and DEP molecules. Besides, the nitrogen atom of the amino group and the oxygen atom of the carbonyl group on the cross-linked adsorbents might also play a role of hydrogen bonding donator and can form hydrogen bonding with the oxygen atom of the ester group of DMP and DEP through the water molecules.

The efficiency of the adsorption process can be predicted by the dimensionless equilibrium parameter $R_{\mathrm{L}}$, which is defined as $R_{\mathrm{L}}=1 / 1+\left(1+K_{\mathrm{L}} C_{\mathrm{o}}\right.$ ) (where $K_{\mathrm{L}}$ and $C_{\mathrm{o}}$ are the Langmuir isotherm model constant and initial aqueous phthalate concentration). As shown in Table $5, R_{L}$ values ranged from 0.09 to 0.58 , and suggested favorable adsorption $\left(0<R_{\mathrm{L}}<1\right)$ of phthalates onto the investigated adsorbent. Also, the Freundlich constants, $K_{\mathrm{F}}$ values, considered as a measure of the adsorption capacity, were higher for DEP than DMP. The reason for the higher affinity could be the higher hydrophobicity of DEP. The Freundlich isotherm indicated that $n$ values ranged from 1.8 to 2.7 , and this implied that the studied adsorption isotherms can be considered nonlinear because the $n$ values were in general outside the $0.95<n<1.05$ range established by Pignatello et al. (2006) as suitable for an isotherm to be considered linear. This can be explained as the manifestation of aromatic $\pi-\pi$ interaction, as Wang, Cook, Tao, and Xing (2007) observed that there was a positive relationship between aromatic carbons and isotherm nonlinearity.

\subsubsection{Effect of temperature and thermodynamics analysis}

The result of the study showed that aqueous temperature had a negative correlation with the adsorption capacities of the adsorbents. Hence, adsorption of phthalates decreased with increment in the temperature of the aqueous media. This is attributed to the reduced interaction of the phthalate molecules with the adsorbent, as a result of increment in the solubility of the phthalates with increment in temperature. Despite having negative correlation with the adsorption capacity, increment in aqueous temperature led to a corresponding increment in the adsorption rate as evidenced in the values of rate constant, $k_{2}$ and initial reaction rate, $h$ (Table S1). This effect may be due to the fact that at higher temperatures, the increase in heat and the subsequent kinetic energy leads to increased mobility of the solute, which results to higher adsorption rates. Also, the observed positive relationship between the temperature and $k_{2}$ as well as temperature and $h$ confirmed the fact that adsorption of phthalate molecules onto the active adsorption sites of the adsorbent surfaces was not the rate limiting step.

The values of the thermodynamic parameters: $\Delta G, \Delta H$, and $\Delta S$ are shown in Table 6 . The $\Delta G$ values for all the adsorbent-adsorbate systems were negative, indicating that the sorption process is 
Table 5

Values of Separation factor (dimensionless equilibrium parameter) $R_{\mathrm{L}}$ for the adsorption of DMP and DEP.

\begin{tabular}{|c|c|c|c|c|c|c|c|}
\hline $\mathrm{DMP} \mathrm{C}_{0}$ & GPP/DMP & GSP/DMP & SPP/DMP & $\mathrm{DEP}_{0}$ & GPP/DMP & GSP/DMP & SPP/DMP \\
\hline 2.41 & 0.55 & 0.57 & 0.47 & 2.40 & 0.56 & 0.58 & 0.47 \\
\hline 4.80 & 0.39 & 0.40 & 0.31 & 4.83 & 0.38 & 0.40 & 0.31 \\
\hline 7.52 & 0.29 & 0.30 & 0.22 & 7.42 & 0.29 & 0.30 & 0.22 \\
\hline 9.88 & 0.23 & 0.25 & 0.18 & 9.69 & 0.24 & 0.25 & 0.18 \\
\hline 12.40 & 0.19 & 0.21 & 0.15 & 12.30 & 0.20 & 0.21 & 0.15 \\
\hline 14.80 & 0.17 & 0.18 & 0.13 & 14.76 & 0.17 & 0.18 & 0.13 \\
\hline 17.43 & 0.15 & 0.16 & 0.11 & 17.23 & 0.15 & 0.16 & 0.11 \\
\hline 19.90 & 0.13 & 0.14 & 0.10 & 20.84 & 0.13 & 0.14 & 0.09 \\
\hline
\end{tabular}

Table 6

Thermodynamic parameters for DMP and DEP adsorption using the studied adsorbents.

\begin{tabular}{|c|c|c|c|c|c|}
\hline \multirow[t]{2}{*}{ Adsorbate/adsorbent } & \multirow[t]{2}{*}{$\Delta H(\mathrm{~kJ} / \mathrm{mol})$} & \multirow[t]{2}{*}{$\Delta S(\mathrm{~kJ} / \mathrm{mol} / \mathrm{K})$} & \multicolumn{3}{|c|}{$\Delta G(\mathrm{~kJ} / \mathrm{mol})$} \\
\hline & & & $298 \mathrm{~K}$ & $318 \mathrm{~K}$ & $338 \mathrm{~K}$ \\
\hline \multicolumn{6}{|l|}{ DMP } \\
\hline GPP & -8.63 & -0.022 & -1.85 & -1.40 & -0.94 \\
\hline GSP & -21.05 & -0.064 & -1.82 & -0.53 & 0.76 \\
\hline SSP & -10.79 & -0.030 & -1.73 & -1.12 & -0.51 \\
\hline \multicolumn{6}{|l|}{ DEP } \\
\hline GPP & -23.76 & -0.071 & -2.37 & -0.94 & 0.50 \\
\hline GSP & -19.43 & -0.058 & -2.06 & -0.90 & 0.27 \\
\hline SSP & -10.26 & -0.028 & -1.91 & -1.35 & -0.79 \\
\hline
\end{tabular}

(a)

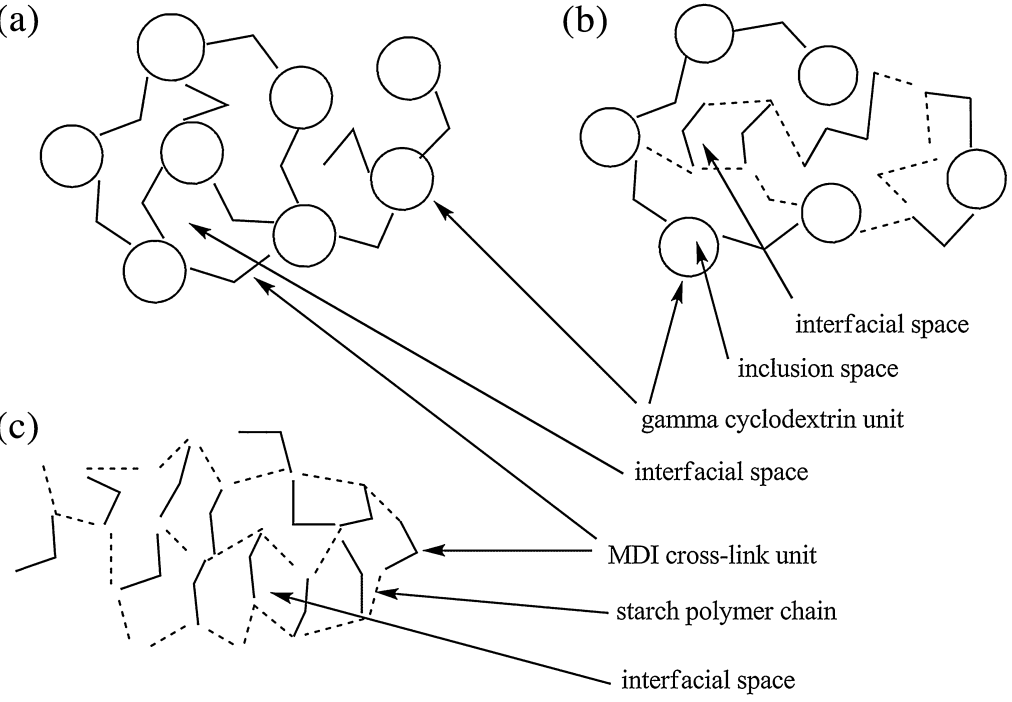

Fig. 7. Schematics of the structure of the polymer adsorbents showing the differences between (a) GPP, (b) GSP, and (c) SPP.

feasible and spontaneous. The adsorptions were exothermic (negative $\Delta H$ values) accompanied by reduced entropy (negative $\Delta S$ values) as the process proceeded to equilibrium.

\subsubsection{Adsorption performance and chemical structural make-up of adsorbents}

Considering the surface and porosity characteristics of the polymer adsorbents as elucidated in Table 1, the expected trend of adsorption capacity was SPP $>$ GPP $>$ GSP. However, both kinetic (Table 2) and isotherm data (Table 4), showed that the batch adsorption capacity (Exp. $q_{e}$ ) of the polymer adsorbents for both DMP and DEP were in the order GPP $>$ GSP $>$ SPP. The observed adsorption trend can be explained to have emanated from the chemical structural make-up of the polymer adsorbents (Fig. 7). Phthalates adsorption on SPP adsorbent occurred on the interfacial spaces as the active adsorption sites, while that of GPP and GSP occurred both on interfacial and inclusion spaces (see adsorption scheme in Fig. S6). The possibility of adsorption in inclusion spaces is predicated on the fact that the volumes guest molecules (adsorbates) were within the range that can be hosted by $\gamma$-cyclodextrin. For instance, the volume of DEP $\left(232.46 \AA^{3}\right)$, as calculated using Density Functional Theorem (Spartan 10v1.1 suite of programmes) is close to that of Benzo[a]anthracene (256.06 $\AA^{3}$ ) which has been reported to be in the size range for $\gamma$-cyclodextrin inclusion (Landy, Mallard, Ponchel, Monflier, \& Fourmentin, 2012). However, due to the fact that GPP has more inclusion spaces than GSP, the contribution of adsorption on inclusion spaces to the entire adsorption was more in GPP than in GSP, hence GPP exhibited higher adsorption capacity than GSP, while SPP had the least.

\section{Conclusion}

The kinetic study revealed that liquid film diffusion is the rate controlling step of phthalate adsorption onto the starch and $\gamma$ cyclodextrin polyurethane polymer adsorbents, while the isotherm study indicated that adsorption of phthalates on external surfaces 
as well as pore surfaces occurred on surface adsorption sites with similar energies. Adsorption is affected by a number of factors, especially phthalate concentrations, adsorbents dose, temperature of aqueous media, and to a less extent $\mathrm{pH}$ and ionic strength. Adsorption was deemed to be complete in reasonable time. Overall, this study demonstrated the treatment of aqueous phthalate pollution by adsorption using $\gamma$-cyclodextrin and starch based polyurethane polymer adsorbents. Considering their good adsorption capacity, ease of synthesis, as well as availability of the polymer precursors at relatively low cost, the adsorbents are promising and cost-effective, and thus recommended for phthalates removal from aqueous environment.

\section{Acknowledgment}

The authors of this work acknowledge the support of The World Academy of Sciences (TWAS) and the Chinese Academy of Sciences (CAS) for providing fellowship to Peter Chukwunonso Okoli (FR number: 3240240233) at the Institute of Geographic Sciences and Natural Resources Research (IGSNRR), CAS, Beijing, China where this research was carried out. The research was financially supported by the One Hundred Talents Program of the Chinese Academy of Sciences, 863 Program (2013AA06A211-2).

\section{Appendix A. Supplementary data}

Supplementary data associated with this article can be found, in the online version, at http://dx.doi.org/10.1016/j.carbpol. 2014.08.016.

\section{References}

Bauer, M. J., \& Herrmann, R. (1997). Estimation of the environmental contamination by phthalic acid esters leaching from household wastes. Science of the Total Environment, 208, 49-57.

Bošnir, J., Puntarić, D., Škes, I., Klarić, M., Šimić, S., \& Zorić, I. (2003). Migration of phthalates from plastic products to model solutions. Collegium Antropologicum, 27(1), 23-30.

Brunauer, S., Emmett, P. H., \& Teller, E. (1938). Adsorption of gases in multimolecular layers. Journal of the American Chemical Society, 60, 309-319.

Chan, H. W., Lau, T. C., Ang, P. O., Wu, M., \& Wong, P. K. (2004). Biosorption of di (2-ethylhexyl) phthalate by seaweed biomass. Journal of Applied Phycology, 16, 263-274.

Chen, C. Y., \& Chung, Y. C. (2006). Removal of phthalate esters from aqueous solutions by chitosan bead. Journal of Environmental Science and Health Part A-Toxic/Hazardous Substances Environmental Engineering, 41, 235-248.

Crini, G. (2005). Recent developments in polysaccharide-based materials used as adsorbents in wastewater treatment. Progress in Polymer Science, 30, 38-70.

Datskevich, E. V. (2009). Removal of phenols from water with cross-linked starch composites. Russian Journal of Applied Chemistry, 82, 2201-2209.

Febrianto, J., Kosasih, A. N., Sunarso, J., Ju, Y. H., Indraswati, N., \& Ismadji, S. (2009). Equilibrium and kinetic studies in adsorption of heavy metals using biosorbent: A summary of recent studies. Journal of Hazardous Materials, 162. 616-645.

Giles, C. H., Macewan, T. H., Nakhwa, S. N., \& Smith, D. (1960). Studies in adsorption. Part XI: A system of classification of solution adsorption isotherms, and its use in diagnosis of adsorption mechanisms and in measurement of specific surface areas of solids. Journal of Chemical Society, 111, 3279-3284.

Guo, L., Li, G., Liu, J., Yin, P., \& Li, Q. (2009). Adsorption of aniline on cross-linked starch sulfate from aqueous solution. Industrial and Engineering Chemistry Research, 48 10657-10663.

Olu-Owolabi, B. I., Diagboya, P. N., \& Adebowale, K. O. (2014). Evaluation of pyrene sorption-desorption on tropical soils. Journal of Environmental Management, 137, $1-9$.

Imam, S. H., Wood, D. F., Abdelwahab, M. A., Chiou, B., Williams, T. G., Glenn, G. M., et al. (2012). Starch: chemistry, microstructure, processing and enzymatic degradation. In J. Ahmed, B. K. Tiwari, S. H. Imam, \& M. A. Rao (Eds.), Starch-based polymeric materials and nanocomposites: chemistry, processing, and applications (pp. 5-32). Boca Raton/London/New York: CRC Press, Taylor and Francis Group.

Julinová, M., \& Slavík, R. (2012). Removal of phthalates from aqueous solution by different adsorbents: A short review. Journal of Environmental Management, 94, 13-24.

Kuniak, L., \& Marchessault, R. H. (1972). Study of the crosslinking reaction between epichlorohydrin and starch. Starch-Stärke, 24, 110-116.

Lagergren, S. (1898). . About the theory of so-called adsorption of soluble substances (24) Handlingar: Kungliga Svenka Vetenskapsakademiens.

Landy, D., Mallard, I., Ponchel, A., Monflier, E., \& Fourmentin, S. (2012). Remediation technologies using cyclodextrins: An overview. Environmental Chemistry Letters, $10,225-237$.

Llompart, M., Garcia-Jares, C., \& Landin, P. (2006). In L. M. L. Nollet (Ed.), Phthalate esters. Chromatographic analysis of the environment, Third edition-revised and expanded (pp. 1103-1153). Boca Raton: CRC Press, Taylor \& Francis.

Mohamed, M. H., Wilson, L. D., \& Headley, J. V. (2010). Determination of host-guest binding sites for $\beta$-cyclodextrin urethane copolymers. Carbohydrate Polymers, 80, 186-196.

Okoli, C. P., Guo, Q. J., \& Adewuyi, G. O. (2014). Application of quantum descriptors for predicting adsorption performance of starch and cyclodextrin adsorbents. Carbohydrate Polymers, 101, 40-49.

Olu-Owolabi, B. I., Diagboya, P. N., \& Ebaddan, W. C. (2012). Mechanism of $\mathrm{Pb}^{2+}$ removal from aqueous solution using a nonliving moss biomass. Chemical Engineering Journal, 195-196, 270-275.

Ozmen, E. Y., \& Yilmaz, M. (2007). Use of $\beta$-cyclodextrin and starch based polymers for sorption of Congo red from aqueous solutions. Journal of Hazardous Materials, $148,303-310$.

Pignatello, J. J., Lu, Y., LeBoeuf, E. J., Huang, W., Song, J., \& Xing, B. (2006). Nonlinear and competitive sorption of apolar compounds in black carbon-free natural organic materials. Journal of Environmental Quality, 35, 1049-1059.

Qiu, H., Lv, L., Pan, B., Zhang, Q. J., Zhang, W. M., \&Zhang, Q. X. (2009). Critical review in adsorption kinetic models. Journal of Zhejiang University of Science A, 10, 716-724.

Simkovic, I., Laszlo, J. A., \& Thompson, A. R. (1996). Preparation of a weakly basic ion exchanger by crosslinking starch with epichlorohydrin in the presence of $\mathrm{NH}_{4} \mathrm{OH}$. Carbohydrate Polymers, 30, 25-30.

Staples, C. A., Peterson, D. R., Parkerton, T. F., \& Adams, W. J. (1997). The environmental fate of phthalate esters: A literature review. Chemosphere, 35, 667-749.

Szejtli, J. (1998). Introduction and general overview of cyclodextrin chemistry. Chemical Reviews, 98, 1743-1753.

Tyler, C., Jobling, S., \& Sumpter, J. P. (1998). Endocrine disruption in wildlife: A critical review of the evidence. CRC Critical Reviews in Toxicology, 28(4), 319-361.

Wang, X., Cook, R., Tao, S., \& Xing, B. (2007). Sorption of organic contaminants by biopolymers: Role of polarity, structure and domain spatial arrangement. Chemosphere, 66, 1476-1484.

Zeng, F. Cui, K., Xie, Z., Wu, L, Liu, M., Sun, G., et al. (2008). Phthalate esters (PAEs): Emerging organic contaminants in agricultural soils in peri-urban areas around Guangzhou, China. Environmental Pollution, 156, 425-434. 